\title{
Sobre el concepto de espacio
}

Alexandre Guida Navarro*

NAVARRO, A.G. Sobre el concepto de espacio. Revista do Museu de Arqueologia e Etnologia, São Paulo, 17: 3-21, 2007.

Resumo: Este artigo oferece ao leitor uma discussão do espaço em várias de suas acepções. Primeiro, discutimos a definição do espaço per se, e como o tema foi trabalhado na literatura, sobretudo na Arqueologia. Em seguida, fazemos uma discussão acerca dos fundamentos da organização espacial. A última parte aborda a discussão do espaço arquitetônico e os elementos culturais responsáveis por sua organização.

Palavras-chave: Espaço - Arqueologia - Cognição - Arquitetura.

\section{El concepto de espacio}

E n este apartado discutimos el concepto de espacio a partir de varias perspectivas, $\mathrm{y}$ las diversas acepciones que este concepto ha adquirido en la literatura. Con ello, ofrecemos al lector las principales vertientes teóricas de cómo se ha trabajado el tema, y las implicaciones al adoptar un estudio de esta naturaleza.

La primera consideración es que el espacio es una de las dimensiones existenciales fundamentales del ser humano, y como tal de su plano vivencial. Este ha sido objeto de estudio y reflexión de la filosofía y las ciencias desde hace mucho tiempo. Por ejemplo, Lao-Tsé, Platón, Aristóteles, Copérnico, Descartes, Locke, Newton y Kant, por citar algunos solamente, definieron este concepto, dándole distintos valores dentro de sus teorías filosóficas.

(*) Instituto de Investigaciones Antropológicas. Universidad Nacional Autónoma de México, UNAM. Doctorado en Antropología. Núcleo de Estudos Estratégicos, UNICAMP. altardesacrificios@yahoo.com.br
Dada la naturaleza de esta investigación, pensamos ser importante ofrecer una interpretación historiográfica acerca del espacio. Aunque es imposible enumerar a todos los investigadores que han tratado el tema, nos parece relevante considerar a algunos de ellos.

Los filósofos griegos, por ejemplo, hicieron del espacio un tema de reflexión. Parménides representó una posición transitoria, al mantener que el espacio, como tal, no podía ser imaginado y que, por lo tanto, no existía. En cambio, Leucipo consideraba el espacio como una realidad, aun cuando no tenía existencia corpórea. Platón llevó más lejos el problema, en el Timeo, al definir la geometría como ciencia del espacio, pero quedó reservado a Aristóteles el desarrollar una teoría del "lugar" (topos). Según él, el espacio era la suma de todos los lugares, un campo dinámico con direcciones y propiedades cualitativas. Su propuesta puede ser considerada como un intento de sistematización del espacio primitivo, pragmático, pero que ya simboliza y preanuncia ciertos conceptos actuales.

Teorías posteriores del espacio se basaron, más que en Aristóteles, en la geometría de Euclides y definieron el espacio como infinito y 
homogéneo: una de las dimensiones básicas del mundo. Lucrecio decía: "Toda la naturaleza se basa en dos cosas; hay cuerpos y hay vacío en el que los cuerpos tienen su lugar y en el que se mueven" (De Rerum Natura I: 420 en NorbergSchulz 1980: 16). La dificultad de concebir la dimensión espacial también se percibe en Vitruvio, quien señala que sólo un arquitecto es capaz de concebir y construir volúmenes y vacíos que no existen más que en su mente (Vitruvius 1983). 1800 años más tarde, Kant todavía consideraba el espacio como una categoría "apriorística" de la inteligencia humana, diferente de la materia e independiente de ella.

En el siglo XVII tuvo lugar un perfeccionamiento sumamente importante de la teoría del espacio euclidiano al introducir el sistema de coordenadas cartesianas u octogonales por Descartes. La última revolución del concepto en las ciencias y filosofía llegó con Einstein (1947:34), que sintetizó los conceptos de espacio existentes en la Física a tres categorías principales:

- Concepto aristotélico del espacio como lugar, refiriéndose a una pequeña porción de la superficie terrestre, con un nombre; el espacio vacío no tiene ningún sentido.

- Concepto de espacio como contenedor, siendo un campo tridimensional, independiente de los objetos materiales, pero el espacio contenido permanece, estando a un nivel superior que lo material; correspondiente a la idea absoluta de espacio de Newton.

- Concepto de espacio cuatridimensional, el espacio relativo.

Las ciencias exactas, en particular, parecen haber descubierto la necesidad de investigar los cambios en la concepción espacial y sus teorías correspondientes en relación con la historia del pensamiento científico y la Física. En el prólogo a Concepts of Space, Max Jammer (1954) escribe que el estudio de la historia del pensamiento científico es esencial para la completa comprensión de los diversos aspectos y realizaciones de la cultura humana. Considera que tal comprensión no se alcanza por el tratamiento de los problemas prioritarios en la historia de los descubrimientos, los detalles de la cronología o incluso la yuxtaposición de todas las historias de las ciencias particulares sino es la historia del pensamiento científico en su más amplia perspectiva contra el fondo de la época la que tiene decisiva importancia para la mente moderna.

Un autor cuyos trabajos son clave para el entendimiento del concepto de espacio es Norberg-Schulz. Este autor señala que la relación del ser humano con el espacio tiene raíces existenciales: "deriva de una necesidad de adquirir relaciones vitales en el medio que lo rodea para aportar sentido y orden a un mundo de acontecimientos y acciones" (Norberg-Schulz 1980: 9). Observa que la mayor parte de las acciones humanas encierran un aspecto espacial entendiéndose por ello que los objetos orientadores están distribuidos según relaciones espaciales, por ejemplo, interior-exterior, lejos-cerca, separadounido. Luego, el ser humano para poder llevar a cabo sus intenciones debe comprender las relaciones espaciales y unificarlas en un concepto espacial. Sin embargo, argumenta que el espacio no es una categoría particular de orientación, sino un aspecto de la orientación cualquiera.

Este autor sigue la discusión subrayando que mientras el espacio pragmático de los animales es una función de instinto innato, el hombre tiene que aprender qué orientación necesita para actuar. Considera que en los lenguajes de las primeras civilizaciones, se encuentran términos que expresan y comunican relaciones espaciales tales como arriba y abajo, delante y detrás, derecha e izquierda. Sin embargo, los términos no son abstractos, sino que hacen referencia al hombre mismo directamente así como el ambiente que lo rodea y expresan su posición en el mundo. Para justificar sus ideas, cita que ciertos idiomas africanos usan la misma palabra para referirse al "ojo" y a "frente a" y que el espacio de los antiguos egipcios estaba determinado por la peculiar geografía del país, y su idioma introdujo las direcciones "agua abajo" y "agua arriba" en lugar de norte y sur. Con esto, el autor deja claro que en ambos casos el concepto cognoscitivo no había sido abstraído de la experiencia de las relaciones espaciales sino que las instituciones espaciales primitivas son orientaciones concretas que hacen referencia a objetos y localidades y, 
por consiguiente, tienen un fuerte referente emocional.

De lo anterior desprende, que el espacio se divide en varios conceptos: espacios físicos concretos (micro, ordinario y macro) y espacios matemáticos abstractos, inventados por el hombre para describir con mayor o menor grado de aproximación a los anteriores. La teoría de la relatividad nos llevaría más allá de esta dicotomía entre espacio y tiempo, "sustituyendo la idea de trozos de materia situadas en el espacio tridimensional, por una serie de acontecimientos en un espacio-tiempo de cuatro dimensiones" (Norberg-Schulz 1980: 9-10).

En el discurso de Norberg-Schulz (1980: 18) también se percibe una vertiente psicológica, y, de hecho, el problema del espacio humano también ha sido estudiado por los psicólogos desde hace un siglo. En este sentido, la experiencia que tiene el hombre del ambiente que le rodea, permite señalar que la percepción del espacio es algo mucho más dinámica que estática. Este autor opina que no se percibe un mundo para todas las personas, sino mundos diferentes que son producto de las motivaciones y experiencias de las gentes. Explica que, en general, "la percepción enfoca suposiciones válidas acerca del medio ambiente que nos rodea y tales suposiciones varían de acuerdo con las situaciones en que participamos" (Norberg-Schulz 1980: 18). Así que, del mismo modo que la Física suele recurrir a una descripción estructural de acontecimientos físicos valiéndose de modelos matemáticos, la psicología debería describir la estructura de los procesos psíquicos por medio de un sistema de conceptos abstractos. Norberg-Schulz (1980: 19) arguye que al igual que los usados en Física, "los antiguos conceptos psicológicos tenían un carácter absoluto, estático, pero con el pasar del tiempo se ha introducido un enfoque más dinámico”. Menciona que las leyes absolutas, por ejemplo, de la psicología de la forma (Gestalt) (nota de pie de página 1) han sido remplazadas por los esquemas más flexibles de Piaget.

Por su parte, Piaget (1966: 5) considera que nuestra conciencia del espacio está basada en esquemas operativos, es decir, experiencia con cosas: "los esquemas espaciales pueden ser de muy distintas clases y el individuo posee más de un esquema capaz de permitirle una percepción satisfactoria de diversas situaciones". Así, los esquemas estarían culturalmente determinados y comprenden propiedades cualitativas resultantes de la necesidad de una orientación afectiva hacia su entorno. Piaget (1966: 6) resume sus investigaciones con estas palabras: "Es completamente evidente que la percepción del espacio implica una construcción gradual y ciertamente no existe ya de antemano al iniciarse el desarrollo mental”.

Podemos percibir en su discurso que el espacio sintético del hombre primitivo ha sido dividido en varias construcciones especializadas de la imaginación, que sirven para nuestra orientación y adaptación a diferentes aspectos del ambiente. Así, que los espacios cognoscitivos, dentro de la dimensión psicológica, tendrían que distinguir entre el espacio perceptivo inmediato y los esquemas de espacio que son más estables. Piaget (1966: 7) arguye que estos últimos están compuestos de "elementos dotados de una cierta invariancia, tales como estructuras elementales universales (arquetipos) y estructuras condicionadas social o culturalmente, y, desde luego, de algunas idiosincrasias personales". Concluye el autor que todo junto forma la imagen del ambiente que recibe el hombre, es decir, un sistema estable de relaciones tridimensionales entre objetos significativos. Esto corresponde a los modelos de la teoría de la Gestalt, que se dedica al conocimiento y a la integración de las diversas partes que forman la personalidad total de cada individuo. Luego, esta terapia facilita un método para descubrir las partes fragmentadas de la personalidad de un individuo, integrarlas y desarrollar un núcleo de confianza en sí mismo. En este sentido, el espacio es una de las variables que torna el individuo miembro de una sociedad.

Fuera del ámbito de las ciencias, la idea del espacio adquiere otras dimensiones. La noción tradicional de espacio está reducida a un problema natural, geográfico, como un mero lugar de residencia o un sitio de explotación. A partir del siglo XIX y bajo la racionalidad burguesa, se considera al espacio como naturaleza a explotar, reduciéndole a su dimensión de territorio: un espacio dominado, que se puede parcelar, medir y vender. Es un concepto que sirve para justificar el uso que se hace de él, en 
consonancia con la propia racionalidad del capitalismo que nacía. Además, dentro del sistema de saber moderno, es interesante la interpretación dada por Criado Boado (1993: 15) en la que el espacio ha estado en descrédito en relación con el tiempo. Citando a Foucault (1984, en Criado Boado 1993: 18), el investigador reflexiona que el espacio es considerado como lo inmóvil, lo muerto, mientras que el tiempo es lo rico y fecundo. Por lo tanto, el espacio quedó en segundo plano.

Norberg-Schulz (1980: 25), al hacer un estudio sobre la noción de espacio, distingue varias concepciones:

- "Espacio pragmático, en donde se desarrolla la acción física; integra al hombre con su ambiente orgánico natural.

- Espacio perceptivo, de acción inmediata; es fundamental para su identidad como persona.

- Espacio existencial, que forma para el ser humano la imagen estable del ambiente que le rodea; le hace pertenecer a una totalidad social y cultural.

- Espacio expresivo o artístico, en el cual se expresa la estructura de su mundo como un real imago mundi. Este tipo de concepto de espacio sistematiza las propiedades posibles de espacios expresivos (dentro de cual se encuentra el espacio arquitectónico).

- Espacio arquitectónico: está directamente relacionado con los esquemas de espacio, del mundo individual y público del hombre. Los esquemas del hombre están creados por influencias recíprocas con espacios arquitectónicos existentes y, cuando éstos resultan confusos o demasiado inestables, se tendrá que cambiar de espacio arquitectónico. Puede ser definido, finalmente, como una concretización del espacio existencial del hombre".

Varios estudios fundamentales sobre el espacio también han sido publicados por filósofos. Los más importantes son los de Merleau-Ponty The Phenomenology of Perception (1962); Otto Friederich Bollnow, Mensch und
Raum (1963); Gaston Bachelard, La Poética del Espacio (1965); el capítulo sobre el espacio en la obra de; $y$, sobre todo, la obra de Martin Heidegger, Ser y Tiempo (1962). Merleau-Ponty critica la superficialidad de ciertas teorías de la psicología de la percepción y demuestra que los signos, que deberían informarnos acerca de la experiencia del espacio, únicamente pueden comunicar la idea de espacio si están ya incluidos en ella y si ya es conocida. Y concluye: “... la profundidad es la más existencial de las dimensiones” (Merleau-Ponty 1962: 256). Para este investigador, el espacio es una de las estructuras que expresan nuestro "estar en el mundo." Sin embargo, fue Heidegger (1962: 31) el primero en mantener que "... la existencia es espacial. No puede disociarse el hombre del espacio. El espacio no es ni un objeto externo ni una experiencia interna. No podemos situar al hombre y al espacio uno al lado del otro...". En Ser y Tiempo, ya subraya el carácter existencial del espacio humano y considera: "'arriba' es lo que está 'en el techo'; 'abajo' es lo que está en el suelo; 'detrás', lo que está a la puerta; todos los 'dóndes' son descubiertos e interpretados con circunspección cuando recorremos nuestros caminos en nuestros quehaceres cotidianos; no son averiguados ni catalogados por la medición observativa del espacio" (Heidegger 1962: 103). Sigue: "Los espacios reciben su esencia de los lugares y no del espacio" (Heidegger 1962: 29). Sobre esta base desarrolla su teoría de "residencia" y revela: "La relación del hombre con los lugares y, a través de ellos, con los espacios, consiste en la residencia. Sólo cuando somos capaces de residir podemos construir. La residencia es la 'propiedad esencial' de la existencia”. (Heidegger 1962: 35).

Para Borrazás et al. (2002: 34), espacialmente, los patrones coordinados de comportamiento son básicos para el ajustamiento personal de todos los seres humanos. Éstos envuelven dimensiones de experiencias fundamentales que son necesarias en la vida social. Consideran que "sin la capacidad de percepción y orientación espacial y de la manipulación de los conceptos espaciales, los seres humanos serían incapaces de coordinar otros aspectos de su comportamiento con los otros miembros que componen su grupo social. El individuo humano está siempre 
provisto de algunos significados culturalmente construidos que constituyen las condiciones que lo hacen capaz de participar con los otros miembros de su grupo de un mundo donde los atributos espaciales son, en parte, conceptualizados y expresados en términos comunes y desarrollados durante el proceso de socialización" (Borrazás et al. 2002: 34). Así que muchos de los componentes adquiridos de la percepción espacial son una función de la cultura a la que el individuo pertenece. Luego, los patrones culturales de diferentes sociedades ofrecen diferentes significados por los cuales las percepciones son desarrolladas, refinadas y ordenadas, es decir los conceptos espaciales dependen de los contextos específicos intencionales propuestos por una determinada cultura.

Como se puede comprobar después de este recorrido por los distintos ámbitos en los que se reflexiona sobre el concepto de espacio, éste ha pasado de ser una noción con un único significado (lugar, espacio tridimensional...), a valorarse de forma multidimensional, considerando tanto su concepción como matriz física como la percepción que el ser humano tiene sobre él y el significado que lleva implícito. Este espacio multidimensional está directamente relacionado con el patrón de racionalidad (lo que LéviStrauss, 1973, llama pensamiento) de la sociedad que lo genera y vive, siendo además la arquitectura el medio más evidente de concretar los conceptos espaciales de esta racionalidad.

En la actualidad, muchos investigadores son partidarios de un método semiológico basado en el estructuralismo francés y las teorías lingüísticas de Noam Chomsky (2000). También se ha intentado desarrollar un método coherente de análisis de la tarea constructiva, especialmente por Christopher Alexander (2000).

\section{Los elementos del espacio}

Tras presentar el concepto de espacio en varios contextos culturales, y las varias vertientes teóricas que lo conforman, seguimos ahora con los elementos que lo definen. A partir de la consulta bibliográfica que hemos hecho, los autores que tratan de definir los elementos del espacio generalmente están de acuerdo que consisten de: centro o nodo, y camino y eje.

\subsection{El centro o nodo}

El primer problema a abordar es la definición espacial de "centro". El centro significa la creación de un lugar, o bien, según la terminología de Lynch (1966), un “nodo". Lynch (1966: 78) introduce también el concepto de landmark para designar "un punto de referencia considerado externo al observador". Los landmarks corresponden a los centros del espacio existencial, aunque a veces su función es más bien la de indicar límites o direcciones.

Según Lynch (1966: 12) la proximidad crea un amontonamiento de elementos, es decir, una concentración de masas. Consiguientemente, encontramos en toda la historia del espacio construido y de la arquitectura, la tendencia a marcar un lugar por medio de una gran masa. Este lugar resume bien lo que se ha definido por "centro". Lynch (1966) considera que tales espacios existen en la naturaleza, por ejemplo, en forma de cavernas. Cita los ritos de iniciación de los "Dogons" que tienen lugar en las cavernas, donde el centro está indicado por una masa concentrada situada en el interior en forma de una piedra erecta de carácter fálico. Así, encontramos los dos símbolos arquitectónicos originales de lugar reunidos: la masa y el centro.

$\mathrm{El}$ autor arguye que en lo que se refiere a la percepción espontánea, el espacio del hombre está subjetivamente centrado. Sin embargo, "el desarrollo de esquemas no sólo significa que la noción de centro está establecida como un medio de organización general, sino que ciertos centros están situados externamente como puntos de referencia en el ambiente circundante" (Lynch 1966: 16). Ejemplifica que en muchos mitos, por ejemplo, el centro del mundo se concretaba con un árbol o un pilar que simbolizaba un axis mundi vertical. Las montañas también eran consideradas como puntos en donde se unían el cielo y la tierra; los antiguos griegos situaban el "ombligo" del mundo (omphalos) en Delfos, en tanto que los romanos consideraban su Capitolio como caput mundi; y para el Islam, la Kaaba todavía es el centro del mundo. 
La aptitud de una masa para servir de “centro" puede ser descrita por el término "concentración”, que es definida como una función de la forma principal y del tratamiento de detalle, $y$, en general, se halla reforzada por una superficie envolvente continua y por la simetría. La concentración se acrecienta también por el aislamiento: "cuando una masa es levantada respecto de su alrededor, existe implícitamente un eje vertical en torno del cual se organiza el espacio (Lynch 1966: 18). Como ejemplo el autor considera que el aislamiento de la Acrópolis de Atenas respecto de la comarca profana que la rodea no sólo realza su sacralidad sino que la convierte en un centro de organización del mundo local entero. Piensa que, en general, la masa es un centro simbólico o ideal, más bien que un lugar real de actividad ya que establece un tope de la extensión horizontal del ambiente que rodea al hombre y hace visible su necesidad de puntos fijos.

Lynch (1966: 27) considera que "se ha atribuido al espacio o al lugar de actividad una de las más antiguas raíces de la masa concentrada, y que el cierre o cerco pueden ser considerados como las primeras tentativas reales de toma de posesión del entorno". Así que mientras que la masa-centro tiene un carácter ideal, abstracto, el cercado tiene fuertes implicaciones sociales, ya que, básicamente, expresa una reunión para un propósito común. Admite que la mayoría de las culturas tiene recintos de esta clase en donde se celebran actos rituales o teatrales. En este sentido, la propiedad arquitectónica esencial sería una delimitación claramente definida que garantiza la protección tanto física como psíquica. Ejemplifica a través del anfiteatro romano cuyo efecto de cerramiento se halla realzado por la forma geométrica y por las líneas de asientos que descienden hacia el centro. La singular distribución de las aberturas también expresarían la función desempeñada por el edificio como centro "social” según Lynch (1966: 28).

\subsection{Camino y eje}

Lynch (1966: 47) define los caminos como "los canales a lo largo de los cuales se mueve el observador habitual, ocasional o potencialmen- te." Norberg-Schulz (1980: 71) añade: "donde se mueve idealmente." Éste considera que el eje organizador, en efecto, no es necesariamente el de un movimiento real; más bien representa una dirección simbólica que unifica cierto número de elementos entre sí y muchas veces los relaciona para formar un todo más amplio. Así, el camino real y el eje son idénticos y pueden poseer componentes horizontales y verticales.

En general, la definición de un camino o de un eje está basada en el principio de continuidad de la Gestalty en una cierta semejanza de los elementos de masa o de espacio que toman parte de la “composición”. Lynch (1966: 43) menciona que "estas unidades se encuentran en la arquitectura popular de todas las partes del mundo y están ordinariamente determinadas por factores geográficos, lo mismo que por tipos particulares de obras y comunicaciones". Sin embargo, señala que en el antiguo Egipto el camino llegó a tener una forma simbólica predominante. Afirma que las tumbas-templo del antiguo imperio (las mastabas) son caminos arquitectónicos consistentes en largos corredores que conducen desde el edificio de recepción junto al Nilo hasta el templo principal al pie de la pirámide. En el templo de la reina Hatshepsut, junto a Deir-el-Bahari, el tema está aún más explícito porque el camino se ha convertido en un eje que divide el edificio en dos partes simétricas. La propia montaña desempeña el papel de la pirámide. En la antigüedad pueden ser halladas otras estructuras del tipo camino, como la Avenida de los Muertos en Teotihuacan o el eje este-oeste del sitio de Dzibilchaltún.

Por su parte, Schwarz (1958) afirma que el carácter de un camino está determinado por la relación de lugares. Argumenta que como todo lugar vive de la tensión entre fuerzas centrípetas y centrífugas, lugar y camino son necesarios y mutuamente dependientes uno de otro. Por ejemplo, en la primitiva iglesia cristiana el espacio interior es un "lugar" aparte y diferente del mundo exterior, pero es interpretado como camino. Semejante a una calle romana flanqueada de columnas, la nave conduce hacia el altar situado en el ábside expresando que el camino es la esencia de la existencia.

Sin embargo, el tema del camino sagrado halló su más espléndida interpretación en las 
iglesias góticas. La continuidad en profundidad ya no fue simplemente expresada por columnas o pilares laterales sino por el entrelazado de nervaduras de las bóvedas, cuyo complejo movimiento busca constantemente un lugar de reposo. En este sentido, el camino es una alegoría de la naturaleza, en la que el hombre toma parte por medio de la arquitectura, la escultura y la pintura.

Por otro lado, en los parques barrocos la toma de posesión de la naturaleza culmina el diseño de los caminos. Por ejemplo, en la ciudad de Santiago de Compostela, al noroeste de España, las torres de las iglesias crean focos o remates sumamente impresionantes al ser vistas desde muchas de las calles de la parte vieja de la ciudad, en el punto central de las mismas, creando así un ambiente de escenografía para el observador en relación con su entorno.

Un ejemplo diferente es la estrella que irradia desde un centro, desarrollada por arquitectos franceses durante el siglo XVII. En este sentido llama la atención que en el Paris de 1740, y en su alrededor, se muestra un sistema de centros y carreteras radiales que transforman el paisaje en una red de caminos, expresión de un nuevo sentimiento existencial, de apertura y expansión (Lynch 1966). En cambio, hoy en día la red de autovías es una mera infraestructura que no conduce nunca a una meta clara y sólo lo atraviesa todo.

Un puente también puede ser una variedad especial de camino, y un río puede servir simultáneamente para separar y para unificar (Lynch 1966: 63). Considera que un puente divide el terreno pero, a la vez, define un espacio que es común a ambas orillas: "el efecto de unificación está ordinariamente reforzado por el terreno, que desciende en pendiente hacia el agua y por el río, al servir como vía de comunicación” (ibid.). Así que el puente hace posible que el transeúnte tome posesión del "río-espacio". Aquí éste se siente fuera y dentro, libre y protegido al mismo tiempo, moviéndose hacia atrás y hacia delante entre dos regiones diferentes aunque pertenecen a una misma totalidad. Ejemplifica que dichas tensiones están bellamente expresadas en el puente de Carlos, en la ciudad checa de Praga (1353). Construido sobre los restos de otra estructura antigua, tiene un trazado curvo muy poco común que viene a resultar como una continuación de las estrechas y sinuosas calles de ambos lados. Además, Lynch (1966: 64) arguye que el puente de Carlos está “'poblado' de estatuas que hacen de él un verdadero centro cívico, es decir, de interacción social entre sus habitantes y los turistas que visitan la ciudad".

Otros investigadores como Baker (1998: 24) y Ching (1995: 18) señalan que las cajas de escaleras y los tramos urbanos de escaleras son caminos de otro tipo. Subrayan que, construidos para ganar una diferencia de niveles, son básicamente verticales. Dando una sensación de victoria sobre la gravedad, tienen un contenido fuertemente expresivo. Dichos autores exponen que las cajas de escalera en espiral siempre han ejercido una especial fascinación al provocar la sensación de elevarse a lo largo del eje vertical; las escaleras urbanas han servido frecuentemente de eslabón de enlace entre un santuario en su cima y una plaza atestada en la base, concretando así la transición de un nivel existencial a otro.

\subsection{Los espacios rurales y urbanos}

Al considerar el espacio como nivel existencial, diversos autores, entre ellos Norberg Schulz (1980), Bender (1993), Baker (1998), Blanton (1994), Rapoport (1990), Ching (1995) y Hillier (1996) subrayan que los paisajes rural y urbano son sus principales variables. Vamos a definir cada uno de ellos.

Desde la antigüedad el hombre ha intentado incidir en las formas de "su" paisaje con la finalidad de transformarlas y encajarlas en su imagen del mundo. Bender (1993: 34) apunta que las pirámides de Egipto constituyen una cordillera artificial que define el límite del espacio "civilizado" a lo largo del Nilo, mientras que más al sur, en Tebas, donde hay montañas reales que cumplen esta misma finalidad, las pirámides eran innecesarias.

Por su parte, Rapoport (1990: 72) señala que el hombre, por medio de sus obras, expresa la capacidad de modelar el paisaje. Observa que como en su vida se desarrolla una acción mutua entre él y la campiña, sus instalaciones suelen articular lugares de la naturaleza. Considera que 
"el nivel de la campiña también puede ser determinado por creencias concretadas a través de la historia por fortificaciones, por una parte, y santuarios, por otra; estructuras que expresan los dos aspectos básicos de la orientación del hombre: seguridad física e identidad religiosa" (Rapoport 1990: 73). Cita al paisaje rural europeo, que estuvo determinado por estos dos elementos: el poderoso castillo y el lejano y divino santuario, que, en algunas ocasiones, estuvieron unificados, como el famoso monte de St. Michel que pretendió simbolizar la ciudad fortificada de Jerusalén.

Hillier (1996: 54) atribuye a los periodos del Renacimiento y del Barroco una tendencia a la geometrización del paisaje, que "empezó por conducir a la creación de pequeñas zonas campestres que contrastaban con la naturaleza virgen alrededor". Posteriormente fue intentada una fusión de las formas artificiales con la naturaleza, hasta que en los parques barrocos se realizó una toma de posesión aparentemente ilimitada gracias a un sistema de caminos organizados geométricamente.

Con relación a los espacios urbanos, Norberg-Schulz (1980: 90) sostiene que los elementos básicos de la ciudad "son el distrito, considerado como una aglomeración o grupo de construcciones más o menos bien definidos; la calle, que es básicamente una formación lineal, y la plaza, que es un cercado". Sin embargo, advierte que esta definición ha sido reemplazada hoy en día por una distribución dispersa de edificios de la ciudad. Las circunstancias topográficas, tales como el curso de un río que divide la ciudad pueden, por ejemplo, contribuir a la definición de diferentes distritos según el autor. Arguye que si suprimimos el río en Londres, París o Roma, la formación de una imagen de la ciudad sería mucho más difícil.

Con relación a la calle, Blanton (1994: 100) observa que en el pasado ésta era la forma condensada del distrito y de la ciudad como un todo. Considera que actualmente se ha perdido el concepto de calle como consecuencia de la ordinaria dispersión de los edificios y el creciente tráfico motorizado. Piensa que, en general, la forma espacial de la calle puede ser definida como longitudinal, pero esto no implica que tenga que ser rectilínea; en las ciudades del pasado los ángulos oblicuos y las líneas curvas creaban una "perspectiva cerrada" que daba vida al panorama.

La plaza, finalmente, es el elemento más distintivo de la estructura urbana (Norberg Schulz 1980; Bender, 1993; Ching 1998). Como lugar claramente delimitado, es el más fácilmente imaginable y representa una meta para el movimiento. Paul Zucker (1959: 1) la ha caracterizado como "lugar psicológico dentro del paisaje cívico". Según Ching (1995: 33) en los mapas de las antiguas ciudades "éstas aparecen como un organismo de conjunto, y la plaza del mercado como núcleo o corazón de tal organismo". Considera que sólo cuando se llegaba a la plaza principal se había realmente "llegado" y en las ciudades más antiguas las calles conducían a este punto focal de una manera natural. Según Norberg Schulz (1980), en Nueva York la Times Square y la Washington Square sirven de puntos focales.

Todos los autores citados están de acuerdo en que incluso en las pronunciadas condiciones "modernas", la plaza satisface las necesidades humanas básicas. La plaza está determinada por los mismos factores formales que la calle, con la diferencia de que los edificios deben formar una continuidad "alrededor" del espacio.

Según su forma, Ching (1995: 75) considera que muchas plazas aparecen compuestas de diferentes “zonas", lo que facilita la presencia simultánea de varias actividades. Tales subdivisiones se acentuarían introduciendo elementos como fuentes o monumentos, articulando el pavimento, o incluso situando los edificios completos en el espacio. Así que "la plaza proporciona la perspectiva necesaria para admirar edificios principales de la ciudad cuyas funciones como hitos físicos y psicológicos quedan así acentuadas. Quitar importantes edificios públicos del núcleo de la estructura urbana es destruirla" (Ching 1995: 78).

Según Baker (1998: 22) la plaza está marcada por un contraste de dimensiones que causa "la experiencia visual de la ciudad". Argumenta que, no obstante, las diferentes dimensiones deben estar relacionadas entre sí en tal forma que podamos identificarnos con el 
complejo total. Esto se consigue, según el autor, cuando los edificios públicos aparecen como variaciones particularmente relevantes de los temas presentes en las casas sencillas.

Para Bender (1993: 43) "las ciudades están con frecuencia geometrizadas, pero la geometrización difícilmente se percibe como tal y más bien contribuye a la imagen de ciertas propiedades topológicas (tales como textura de la región, continuidad de los caminos o cierre de la plaza)". Luego, los niveles del espacio formarían una totalidad estructurada que corresponde a la estructura del espacio existencial.

Según Bailey (1990: 63), como la identidad del hombre se da con relación a la totalidad del espacio existencial, "todos los niveles del espacio arquitectónico tienen que tener su identidad definida, sin lo cual, la imagen que el hombre se forma de su ambiente sería confusa y su propia identidad personal amenazada". El nivel del paisaje rural es considerado como una base que contiene todas las estructuras particulares de la vida y la acción: su identidad depende de una continuidad general. El nivel urbano, en cambio, se distingue por su concentración y densidad y que los hombres se reúnen en la ciudad y su identidad depende de esa coexistencia, según el autor.

Para Criado Boado (1999) el "campo" del espacio existencial está representado (concretizado) por un aspecto arquitectónico correspondiente. Cuando intervienen los campos de los diferentes niveles, se forma una totalidad muy compleja. Así que el espacio puede contener diferentes estructuras, a diferentes niveles y la influencia recíproca entre ellas varia a lo largo del tiempo. Luego, el nivel urbano o agrupación de edificios “está estructurado topológicamente sobre las bases de cierre y proximidad, mientras que los propios edificios están geometrizados y cuidadosamente articulados" (Criado Boado 1999: 12).

Para Bourdieu (1990: 13) en la arquitectura del Renacimiento se intentó una interfase aún más completa de los niveles basando todos los edificios sobre las mismas sencillas unidades geométricas e integrándolos en un espacio euclidiano. En este caso, se encuentra la misma estructura en todos los niveles e incluso a la naturaleza se le ha dado forma geométrica en los jardines ceremoniosos. Este ideal "clásico" de estructura uniforme, que expresa la imagen de un universo ordenado y armonioso, ha continuado obsesionando a los arquitectos hoy en día.

Según Low (1995: 758) las plazas también son "importantes representaciones sociales del espacio jerárquico". Considera que cualquier forma espacial es generada por fuerzas sociopolíticas conflictivas, en donde implicaciones políticas están por detrás de las construcciones arquitectónicas. Observa que el diseño de un espacio urbano refleja la acción política del Estado. De ello, el espacio es la metáfora del dominio de un grupo sobre otro. En su estudio sobre las plazas concluye que las ciudades construidas durante el proceso de colonización en América española evidencian relaciones de poder para subordinar a la comunidad, ya que fueron construidas encima de construcciones indígenas, en especial templos y lugares asociados con "lo sagrado".

Para Zucker (1959: 32), una topología formal de plazas está compuesta de cuatro elementos: (1) la plaza cerrada, en la que el espacio (rectangular, circular o cuadrangular) está cerrado por formas de estructuras recurrentes; (2) la plaza dominada, en la que el axis espacial se centra en una estructura dominante, focal; (3) plaza nuclear, en la que el núcleo de un espacio abierto es creado por un monumento central o una fuente; y, por último, (4) las plazas agrupadas, que son múltiples espacios contiguos, físicamente unidos por caminos artificiales y visualmente articulados por una estructura dominante en el espacio.

Sin embargo, Moore (1996: 790) llama la atención acerca de la función de las plazas, que cualesquiera que sean, siempre estarán de acuerdo con el sentido de la interacción humana. Según el, "las plazas son definidas culturalmente como lugares de diversas interacciones públicas, y con muchos significados semiológicos, que pueden ser sagrados o mundanos, según su uso para las procesiones, comercio, ejecuciones, consagraciones, juegos, fiestas, o como lugares de encuentro para charlar" (Moore: 1996: 792). Aunque distintas actividades pueden ser llevadas a cabo en la plaza, no todos los espacios abiertos tuvieron el mismo diseño y/o las mismas intenciones. El tamaño, la ubicación, las maneras de acceder 
hacia ellas son parte de diferentes contextos de interacción humana, y por lo mismo, tienen diferentes significados culturales dentro de cada sociedad según el autor.

Hay también quienes encuentran en las plazas un significado de identidad cultural (Rautman 2000). Serían espacios en donde se llevan a cabo "actividades religiosas o laicas que otorgan a los individuos que componen la sociedad un grado de auto-identificación que los lleva a considerarse como un grupo único" (Rautman 2000: 276). Considera que este proceso cultural suele ocurrir en sociedades que pasan por periodos de reorganización interna de los espacios y la redistribución de la población regional.

\section{La organización del espacio}

En este apartado discutimos la organización del espacio. Uno de los aspectos más recurrentes en la literatura es su carácter cognitivo. Entre los exponentes más importantes en este sentido se encuentran Parker Pearson y Richards (1994).

En sus escritos, estos autores señalan que el espacio comprende y expresa ciertos principios de orden y clasificación. Así que "como un medio cultural construido, el espacio es un contexto definido en el cual las personas realizan actividades específicas en tiempos específicos" (Parker Pearson y Richards 1994: 6). Por esa razón, afirman que el significado espacial es obtenido a través de las prácticas sociales. Por lo tanto, los medios ocupados son pensados antes de ser construidos y existen en términos de nuestras acciones y significados: "el espacio arquitectónico puede ser definido como la concretización del espacio existencial, es decir, los elementos del paisaje son construidos culturalmente y transformados dentro de sus marcadores materiales y permanentes autenticando la historia, la experiencia, y por extensión la cultura de un grupo" (Parker Pearson y Richards 1994: 7).

Según los autores, la determinación de los espacios puede tener profundos efectos sobre otros aspectos de la cultura y sociedad. Tal fenómeno juega un papel decisivo en la constitución de la sociedad una vez que el espacio puede ser definido como símbolo y la arquitectura como medio simbólico. En dicha concepción, empero, los significados que son atribuidos a los lugares y al orden social no necesariamente son estables o invariables, ya que sirven para invocar el contexto de uso corriente en la sociedad. Los significados sociales se adhieren a la estructura espacial cuando media la actividad humana, es decir, personas activamente dan sus significados al espacio y entones actúan sobre esos significados dados. Por lo mismo todas las sociedades humanas han implantado en sus asentamientos algún nivel de estructuración espacial en el sentido de organizar el caos.

Estudios llevados a cabo por científicos de la teoría del comportamiento (Sanders 1990; Reid 1989; Watson 1970) han indicado que la organización del espacio es un factor vital para el bienestar psicológico de los hombres. La definición de espacio, en este sentido, es la manera de transformar y contener el entorno ambiental a través de ideologías culturales específicas. Luego, el orden espacial creado reflejará y reforzará las relaciones entre las personas de aquella cultura.

La artificialidad del medio construido ha sido destacada por muchos investigadores (Rapoport 1969; Kus 1983; Foster 1989; Hingley 1990; Parker Pearson y Richards 1994), no meramente como respuesta a las necesidades del medio, sino como un microcosmo de la sociedad y una herramienta para comprender la estructura social. Sanders (1990: 43) afirma que "the individual behavioural decisions, which reflect more general cultural values are embodied in the formation and use of the built environment. Architecture would, therefore, seem to be one of the most valuable artefacts at the disposal of cultural historians, whether the context is ancient in contemporary societies".

En este sentido, para Parker Pearson y Richards (1994: 12) "la extensión de este valor es dependiente del conocimiento de los valores y creencias de una sociedad". Así que es posible, a través de los estudios antropológicos, percibir como la arquitectura refleja los principios sociales y cosmológicos. Ejemplifican que las anchas casas de los barasana de Amazonia tienen un acceso, destinado solamente a los hombres, a través del este, que da de frente hacia el "río de 
leche" (el Amazonas), y un acceso destinado a las mujeres en el lado opuesto. Luego, "la casa forma un microcosmos de su mundo, en donde el techo representa el cielo, las estacas las montañas, y abajo del suelo el inframundo" (Parker Pearson y Richards 1994: 13). Este ejemplo ilustra una de las razones de por qué el espacio es organizado, incorporando conceptos simbólicos específicos.

Para Kus (1983: 27) el espacio natural transformado por el hombre o el espacio creado por él "refleja los conceptos sobre el universo y sobre la sociedad misma". Debido a que los movimientos de cuerpos celestes se perciben en el espacio, la autora considera que "los hombres se aprovecharon de las propiedades celestes para desarrollar sus cosmovisiones, o sea, interpretaciones culturales de la estructura cosmogónica" (Kus 1983: 27). Añade que muchas veces los asentamientos humanos fueron erigidos y orientados hacia los cuatro rumbos del universo, y que los espacios principales y sagrados se ubican en el centro del asentamiento o en partes elevadas, como réplicas del orden cósmico. Admite que, al mismo tiempo, los centros ceremoniales fueron vinculados con el tiempo, pues el transcurso de los ciclos calendáricos se concebía como un recorrido a lo largo de los rumbos del universo. De este modo, los asentamientos humanos en general constituyen una réplica del mundo, imago mundi, concebidos de acuerdo con las cosmologías de cada cultura.

Otro aspecto importante en la organización del espacio es el concepto de frontera. Sanders (1990: 49) ha afirmado que "human beings are territorial animals. We define space, mark them for specific uses, create visible and invisible boundaries, establish cultural conventions of behaviour towards those boundaries, and will defend the territory against unwanted intrusions".

Las fronteras territoriales pueden tener diferentes formas y ser usadas para diferentes propósitos. Rapoport (1990: 298) ha notado cómo ciertos medios ambientes sencillos pueden estar altamente divididos conceptualmente. Para el autor estas divisiones dependen de aspectos culturales inherentes de cada sociedad, que pueden influenciar el tipo de comportamiento propio de su cultura. El problema para el arqueólogo sería, entonces, hallar éstas fronteras conceptuales y las actividades llevadas a cabo en el sitio, a través, por ejemplo, de la distribución de los artefactos intrasitio.

Los mismos conceptos pueden aplicarse a las fronteras físicas, las cuales marcan la separación de diferentes esferas funcionales que, a su vez, pueden indicar los modos de comportamiento. Por ejemplo, las casas Swahili, descritas por Donley-Reid (1990: 120) fueron divididas internamente dentro de áreas que fueron asignadas a individuos de diferentes niveles sociales. El nivel más inferior de la casa fue usado solamente por esclavas domésticas como área de almacenamiento, mientras que el nivel superior fue utilizado por los que nacieron libres. De esta manera, la frontera entre los dos niveles tiene conceptos culturales propios que afectan el comportamiento de las personas, así como refuerza el significado de su orden social específico. Aunque las fronteras puedan tener muchos propósitos, como la defensa, administración o residencia, "la función subyacente es aquella que clasifica los lugares y controla los accesos. En el último caso, los puntos de transición (entrada/ salida) son de particular importancia, ya que regulan el movimiento de un área hacia otra" (Donley-Reid 1990: 116).

Otro aspecto importante en la organización del espacio es la relación del hombre con el medio ambiente y su entorno. Para Pearson Parker y Richards (1994) los principios de organización espacial pueden derivarse de ello. Por ejemplo, la estructuración concéntrica del espacio en un centro y una periferia (o un conjunto de zonas concéntricas) y la organización diamétrica de acuerdo con uno o más ejes (por ejemplo los cuatro puntos cardinales) también son elementos comunes de un sistema subordinado a reglas y a convenciones. Luego, a través de la imposición y articulación de esos varios principios subordinados, los hombres crean el orden social o el cosmos.

Los mismos autores afirman que una de las principales características de una sociedad es su orden, es decir, el sentido de coherencia de cada aspecto de la vida que deriva de un conocimiento compartido de informaciones de cada evento. La incorporación de cualquier o todos esos códigos subordinados dentro de la organización física del medio ambiente construido por los hombres 
según los autores es nombrada "arquitectura sagrada”. El principal propósito de la arquitectura sagrada - y de toda la arquitectura de tipo templo, palacio y tumba - sería su lugar como frontera entre el pensamiento humano acerca de Dios y el universo.

Aquí sería interesante relacionar el espacio y el medio ambiente como factores culturales, es decir, el medio ambiente refleja la cultura y los procesos culturales para organizar el espacio (Rapoport 1972). Este autor piensa que los medios ambientes son producto de la acción humana y de la cultura. Nunca son caóticos, siempre hay una organización intencional. El autor propone un conjunto de cuestiones para inferir los significados culturales del espacio y del paisaje:

- ¿Cuáles características de las personas como miembros de grupos sociales, o como individuos, afectan la construcción del medio ambiente?

- ¿Cuáles son los efectos de los aspectos del medio ambiente sobre los individuos y grupos? ¿cuáles son las circunstancias y condiciones de estos efectos?

- ¿Cuáles son las dinámicas de funcionamiento de estos mecanismos de interacción? ¿Responder a estas cuestiones acaso facilitaría la comprensión de los fenómenos en los procesos culturales?

Así que el espacio está dispuesto dentro de sistemas en que las actividades pretendidas están organizadas sistemáticamente dentro del paisaje, es decir, hay un orden secuencial para la función de la actividad que se pretende llevar a cabo en dicho espacio. El uso sistemático del espacio, basado en reglas definidas por un grupo y significados que son organizados culturalmente genera la organización del mismo. De esta manera, Rapoport (1972: 48) aclara que el espacio es "culturalmente clasificado y socialmente regulado, es decir, resulta cambiante, dinámico, en lo cual el medio ambiente construido o el paisaje es la expresión física de la organización del espacio que es visible, observable".

Podemos percibir, de este modo, que la organización del espacio obedece a criterios cognitivos, las diferentes maneras en las que el espacio es organizado reflejan las expresiones físicas de un esquema de la mente humana en sociedad. La organización del espacio puede ser estudiada en términos de status, poder, acciones sociales de un grupo y en términos de significado cultural expresada en el mito, ritual, culto y simbolismo. Rapoport (1969) ha afirmado que estos factores son sugeridos como las principales razones de los orígenes de las ciudades. El espacio también puede tener un carácter sagrado que sirvió para legitimar las formas de su organización que están basadas en aspectos del medio ambiente, paisaje y factores culturales.

Explorando la cognición del espacio, Rapoport (1990: 12) considera que se expresa a través del medio ambiente y del paisaje y pueden ser decodificados y reproducidos como "mapas cognitivos o mentales”. En este sentido, sostiene que tres aspectos fundamentales envuelven la concepción de la organización del espacio:

- La clasificación del espacio refleja un esquema cognitivo, códigos y reglas culturales.

- Las reglas envuelven un cierto grado de control por los individuos o grupos y aluden a un control del comportamiento.

- El espacio es una forma de comunicación de diferentes naturalezas y sirve para la manifestación de aspectos culturales específicos, como un culto.

Finalmente, el espacio es organizado jerárquicamente y es la expresión de las instituciones sociales, de los sistemas, de los grupos y del esquema cognitivo, y sugiere que es a través de la investigación acerca del contexto específico que se pueden comprender las diferentes maneras en las cuales el espacio está organizado. Luego, la organización del espacio sería una expresión del medio ambiente, del paisaje y de la cultura material y es indisociable de los aspectos de vida humana. La organización espacial refleja, refuerza y guía la comunicación entre los miembros de un grupo humano a través de los significados culturales trasmitidos como códigos dentro de una sociedad.

A partir de todas estas discusiones, podemos observar una preocupación por parte de los investigadores mencionados de resaltar que el 
espacio es un elemento de la cognición humana y expresa principios de orden y clasificación según las necesidades de las acciones sociales. El espacio cumple con la función de expresar ideas plasmadas en un lugar específico para servir a alguna actividad humana especifica. La noción de una organización del espacio implica dos condiciones que necesitan ser satisfechas con relación a la imposición humana sobre la naturaleza y la reorganización de su espacio: la primera implica la conceptualización de ideas en cuanto a un orden o sistema cuya calidad es la capacidad humana de diferenciar este orden con relación a las imposiciones de la naturaleza; la segunda, la formulación de medios que viabilicen los conceptos espaciales a ser creados y su relación con el medio. Puede concluirse, entonces, que la noción de una organización espacial implica un fin o concepto predeterminado, cuya materialidad es resultado de un proceso cognitivo.

Para terminar esta sección quisiéramos discutir las posibles razones para la diferenciación del espacio en cuanto a los factores culturales que conllevan a su organización, siendo que está presente en todas las sociedades en cierto grado.

La división del espacio evidencia una serie de factores primarios, todos relacionados con la necesidad humana del orden. Las cosmologías, que ayudan a moldear este orden, pueden tener una fuerte influencia en el uso del espacio, como en el ejemplo de las casas Barasna antes mencionado (Parker Pearson y Richards 1994: 13). Esto es pronunciado especialmente en los lugares sagrados, que en muchas culturas son vistos como microcosmos terrestres del dominio divino (e.g. templos hindus). Algunos investigadores como Raglan (1964) y Highlands (1990) argumentan que las ideologías religiosas se originan en las viviendas domésticas, mientras que Rapoport (1969: 40) piensa que "hay una diferencia considerable entre las edificaciones que incorporan aspectos cosmológicos y las edificaciones construidas especialmente para propósitos religiosos”. El problema está, de este modo, en la dificultad de identificar los límites de un espacio en cuanto su propiedad sagrada y profana. Por ejemplo, dentro de las casas Swahili, mencionadas anteriormente (DonleyReid 1990: 121), hay un cuarto central conocido como ndani que, además de ser el espacio secular de las mujeres, también fue utilizado como espacio ritual. No hay, en este caso, una separación pronunciada del espacio sagrado y profano, y la ausencia de esta diferenciación no es poco común en sociedades donde la vida secular y religiosa estuvieron tan estrechamente ligadas.

Las cosmologías pueden ser vistas como un efecto sobre el orden espacial de muchas estructuras para reforzar la jerarquía social. Para Fairclough (1992: 348) el principio de la organización espacial es el que "the fundamental structures of a society condition the ways in which groups negotiate rights over space within a building". Es decir, los principios subyacentes de una cultura son un factor vital que influye en el uso de sus espacios. La diferenciación espacial es muchas veces utilizada para establecer y mantener las divisiones y jerarquías dentro de una sociedad, a través del género, edad o nivel social o jerárquico. Personas de gran influencia social pueden trasformar el orden espacial dentro de mecanismos neumónicos que reflejan su posición superior afirmando su poder (Donley-Reid 1990: 115). Así que hay un problema en determinar cómo las estructuras sociales pueden ser reconocidas visualmente en el orden espacial.

Los autores están de acuerdo que otra razón primaria para la diferenciación del espacio se relaciona con la necesidad de definir las áreas de actividad. Las zonas funcionales son muchas veces separadas una de la otra, aunque la frontera entre ellas pueda ser solamente conceptual en naturaleza. Esta necesidad de separar los espacios viene del deseo básico humano de establecer el orden del espacio natural que los rodea. Aunque la relación entre los hombres y el orden espacial es dinámica, el uso específico del espacio puede variar considerablemente a lo largo del tiempo. El analizar áreas de actividad particulares en el registro arqueológico puede ser algo bien problemático como lo puede ser también el espacio ya que, consecuentemente, puede ser utilizado por una variedad de personas para diferentes propósitos. Según Kent (1990: 4) tales áreas podrían evidenciar, a través del registro arqueológico, "una multifuncionalidad, especialmente si hay un breve periodo de cesación entre las actividades". Este problema es más pronunciado cuando se trata de 
espacios públicos, en donde muchos diferentes grupos pudieron utilizar el mismo espacio por un cierto periodo de tiempo.

Rapoport (1990: 11) ha afirmado que las actividades llevadas a cabo en el espacio son expresiones directas del estilo de vida de una cultura, y las dividió en cuatro categorías:

1 - La actividad en sí misma.

2 - Cómo es realizada esta actividad.

3 - Cómo la actividad es asociada con otras actividades, y cómo estas son combinadas dentro de un sistema de actividades.

4 - El significado de esta actividad.

Sus presupuestos enfatizan esencialmente cómo la misma actividad puede manifestarse de diferentes maneras en el mismo espacio, de acuerdo con su contexto específico. Desde la categoría 1 hasta la 4 hay un gran grado de variación cultural, terminando en el significado de la actividad que puede variar mucho dentro de una misma cultura. Esto podría ayudar a explicar la gran variedad de las formas físicas usadas para realizar el mismo conjunto de actividades. La relación entre las actividades y el medio ambiente ha sido el centro de muchos debates (Rapoport 1969, 1990; Donley-Reid 1990; Ciolek-Torrello 1984). La discusión se ha centrado principalmente en saber si es posible identificar la función de una determinada actividad en el espacio a través de las formas arquitectónicas, y si sus características pueden indicar el tipo de actividades que se llevaron a cabo en ella.

Según Smith (2001: 19) la interpretación de estructuras puramente a través de datos morfológicos "presenta dificultades al analizar centros de culto en Gran Bretaña durante la Edad del Hierro". Observa que en este tipo de análisis se deben considerar otros elementos que corroboren el conocimiento de la cultura, como los escritos o la iconografía, junto con los tipos arquitectónicos funcionales específicos. En este tipo de estudio, cita a Forcey 1998 (en Smith 2001: 19) y su análisis de la forma del templo celta-romano.

\section{El estudio del espacio arquitectónico}

El estudio del espacio arquitectónico, relacionado a la arquitectura moderna, nace en las últimas décadas del siglo XIX con el art nouveau, rompiendo con el eclecticismo decimonónico dominante y destacando la fusión de ornamentación y construcción del edificio. Es el primer movimiento que visualizó la nueva conciencia de la abstracción espacial, surgiendo así por primera vez la idea de espacio en cuanto idea arquitectónica (Mañana Borrazás et al. 2002).

A partir de ese momento, arquitectos e historiadores del arte empiezan a considerar la arquitectura como un concepto fundamental dentro del espacio. Lao-Tsé es uno de los filósofos antiguos que más notablemente ha influido en este cambio de concepto. En su teoría, que trata del espacio interior de las construcciones, desarrollada hacia el 550 a.C., estableció los cimientos del principio filosófico y fenomenológico de la polaridad, uniendo en un solo concepto el Ser y No Ser.

En el estudio del concepto de espacio arquitectónico destacan las teorías de los siguientes historiadores del arte y arquitectos. Para Schmarsow (en Norberg-Schulz 1980: 46) "la arquitectura se genera a partir del cuerpo humano, de modo que lo que busca éste es su propio movimiento y el de sus extensiones en el espacio, dependiente su carácter funcionalista”. Distingue tres modalidades de espacios: el táctil, el móvil y el visual, con lo que incorpora los sentidos en simultáneas y sucesivas experiencias en el espacio y en el tiempo. Hildebrand (en Norberg-Schulz 1980: 48) percibe el espacio arquitectónico de dos maneras: "a través de la visión pura (imágenes en reposo) y la visión cinética (imágenes recibidas con el espectador en movimiento)". También han sido fundamentales las teorías cubistas, que a partir de la teoría de la relatividad de Einstein (1947) añaden el concepto de la cuarta dimensión, que se adquiere con el movimiento del cuerpo (frente a la dimensión euclidiana, que es estática).

Sin embargo, uno de los mayores representantes de la arquitectura moderna, Le Corbusier (1967: 56), rechaza en un principio esta cuarta dimensión, alegando que "el hombre sólo puede percibir tres, pero finalmente la rehabilitó llamándolo espacio inefable o 'estado emocio- 
nal por el que se experimentan espacios definidos por series armónicas', lo que deriva en una gran consideración en la disposición del volumen y al orden de la circulación en los edificios". En todas estas teorías, la percepción de los espacios, siendo el movimiento una de sus claves, se perfila como fundamental en el estudio de la arquitectura.

Uno de los autores fundamentales en la actualización del concepto de espacio arquitectónico ha sido S. Giedion $(1980,1988)$, para quien el proceso por el cual una imagen espacial puede ser transpuesta a la esfera emocional es expresado por el concepto espacial: "Proporciona información acerca de la relación entre el hombre y lo que lo rodea. Es la expresión espiritual acerca de la realidad que se halla frente a él. El mundo situado ante él es modificado por su presencia; le obliga a proyectar gráficamente su propia posición si desea relacionarse con él" (Giedion 1980: 13). Con esta frase el autor resume sus ideas considerando que en el campo de la arquitectura el espacio es experimentado por medio de la observación, en la cual los sentidos de la vista y el tacto están entrelazados.

Según Giedion (1988) la luz también produce la sensación de espacio. Reflexiona que el espacio es aniquilado por la oscuridad. La luz y el espacio, así siendo, son elementos inseparables. Así que si la luz es suprimida, el contenido emocional del espacio arquitectónico desaparece, haciéndose imposible de percibir. La esencia del espacio arquitectónico se halla en la interacción de los elementos que lo limitan.

Por otro lado, los estudios sobre el espacio arquitectónico dependen del concepto de espacio base que se utilice. Norberg-Schulz (1980: 13) distingue dos tipos de estudios:

\section{- "Los que se fundamentan en el} espacio euclidiano, tridimensional, y estudian su gramática: se basan en el desarrollo de modelos de dos o tres dimensiones de carácter geométrico, formando parte ésta de la sintaxis del espacio arquitectónico".

- "Los que tratan de desarrollar una teoría del espacio sobre la base de la psicología de la percepción, sobre todo de las impresiones, sensaciones y estudios de los efectos que se efectúen sobre el ser humano que percibe".

Muchos arquitectos consideran que cualquiera de estos estudios tiene un grado de deficiencia, ya que el primero ha excluido al ser humano, discutiendo la geometría abstracta; $y$, el segundo ha reducido el espacio y la arquitectura a impresiones, olvidándose del espacio como dimensión existencial y como relación entre el hombre y el medio que lo rodea. Para salvar esta carencia, Norberg Schulz (1980: 13-15) propone introducir el concepto de espacio existencial dentro del estudio del espacio arquitectónico, "ya que estudiando únicamente aspectos geométricos o de percepción, se obtendría un análisis parcial, superficial. Sin embargo, si se analizan dentro de un esquema de comprensión más general, cada uno de estos aspectos adquiere una mayor significación. Así, un estudio de la especialidad de una construcción arquitectónica que no está integrado dentro de la racionalidad que la generó, queda mutilado, sin sentido". Esta es precisamente una de las teorías esenciales de las que parte este texto: la asunción de que las actividades que tienen lugar en relación con el espacio están organizadas de forma coherente con la representación ideal del mundo que tiene el grupo social que las realiza, por lo que es fundamental integrar estos análisis, tanto el gramatical como el de la percepción espacial, en un determinado patrón de racionalidad.

Para Norberg-Schulz (1980: 7) la discusión acerca del espacio arquitectónico ha estado dominada por un "ingenuo realismo", disfrazado unas veces de estudios de percepción arquitectónica y otras de geometría tridimensional. En ambos casos se omite el problema básico del espacio arquitectónico como dimensión de la existencia humana, con el resultado de que el concepto de espacio se mira con frecuencia hoy día como anticuado o incluso superfluo. Sobre la base de una teoría del espacio existencial, el espacio arquitectónico puede ser interpretado como una "concretización" de esquemas ambientales o imágenes que son una parte necesaria de la orientación general del hombre o de "su estar en el mundo". 


\section{Agradecimientos}

Agradezco al Dr. Felipe Criado por la invitación en participar de una estancia de investigación en el Laboratorio de Paleoambiente, Patrimonio y Paisaje del Instituto de Estudios
Gallegos Padre Sarmiento, Santiago de Compostela, España. A Bernd Fähmel Beyer, del Instituto de Investigaciones Antropológicas de la UNAM, por la lectura y sugerencias del artículo. Y al Dr. Pedro Paulo Funari, de la UNICAMP, por el constante estímulo y atención.

NAVARRO, A.G. On the concept of space. Revista do Museu de Arqueologia e Etnologia, São Paulo, 17: 3-21, 2007.

Abstract: This article offers a discussion about the space in its several meanings. First, we discuss the definition of the space in itself, and how the theme has been handled in literature, specially in Archaeology. Next we make a discussion about the foundations of spatial organization. The last part addresses the discussion of architectonic space and cultural elements responsible for their organization.

Keywords: Space - Archaeology - Cognition - Architecture.

\section{Referencias bibliográficas}

ALEXANDER, C.

2000 Urbanismo y participación. México: UNAM.

BACHELARD, G.

1965 La poética del espacio. México: UNAM.

BAILEY, D.W.

1990 The Living House: Signifying Continuity. In: Samson, R. (Ed.) The Archaeology of Houses. Edimburgo, Edinburgh University Press: $19-48$.

BAKER, G.H.

1998 Análisis de la forma: urbanismo y arquitectura. México: Gustavo Gili.

BENDER, J. (Ed.)

1993 Landscape, Politics and Perspectives. Oxford: Bergh.

BLANTON, R.

1994 House and Households. Nueva York: Plenun Press.

BOLLNOW, O.F.

1963 Mensch und Raum. Berlim: Möckmühl.

BOURDIEU, P.

1990 Outline of a Theory of Practice. Cambridge: Cambridge University Press.

CHING, F.

1995 Arquitectura: forma, espacio y orden. México: Gustavo Gili.
1998 Diccionario visual de arquitectura. México: Gustavo Gili.

CHOMSKY, N.

2000 New Horizons in the Study of Language and Mind. Cambridge: Cambridge University Press.

CIOLEK-TORRELLO, R.

1984 An Alternative Model of Room Function From Grasshopper Pueblo, Arizona. In: Hietata, H. (Ed.) Intrasite Spatial Analysis in Archaeology. Cambridge, Cambridge University Press: 127-153.

CRIADO BOADO, F.

1993 Limites y posibilidades de la arqueología del paisaje. Sevilla, Spal, 2: 9-55.

1999 Del terreno al espacio: planteamientos y perspectivas para la arqueología del paisaje. CAPA, 6. Santiago de Compostela: Grupo de Investigación en Arqueología da Paisaxe.

DONLEY-REID, L.

1990 A Structuring Structure: the Swahili House. Kent, S. (Ed.) Domestic Architecture and the Use of Space. Cambridge, Cambridge University Press: 114-126.

EINSTEIN, A.

1947 The Problems of Space, Ether and the Field of Physics. Nueva York: Random House. 
FAIRCLOUGH, G.

1992 Meaningful Constructions - Spatial and Functional Analysis of Medieval Buildings.

FOSTER, A. Antiquity, 66: 348-366.

1989 Analysis of Spatial Patterns in Buildings as an Insight Into Social Structure: Examples From the Scottish Atlantis Iron Age. Antiquity, 63: 40-50.

FOUCAULT, M.

1984 Vigilar y castigar. El nacimiento de la prisión. Madrid: Siglo XXI.

\section{GIEDION, S.}

1980 Space, Time and Architecture. Cambridge: Harvard University Press.

1988 El presente eterno: los comienzos de la arquitectura. Madrid: Alianza.

GOODMAN, M.; FORMAN, R.

1986 Landscape Ecology. Nueva York: J. Wiley $\&$ Sons.

HEIDDEGER, $M$.

1962 Ser y Tiempo. Buenos Aires: Alianza.

HIGHLANDS, D.

1990 What's Indigenous? An Essay on Building. In: Turan, M. (Ed.) Vernacular Architecture: Paradigms of Environmental Response. Aldershot, Avebury: 121-142.

HILLIER, B.

1996 Space is the Machine. Cambridge: Cambridge University Press.

HINGLEY, R.

1990 Public and Private Space: Domestic Organisation and Gender Relations Among Iron Age and Romano-British Households. Samson, R. (Ed.) The Social Archaeology of Houses. Edimburgo, Edinburgh University Press: 125-148.

JAMMER, M.

1954 Concepts of Space. Cambridge: Harvard University Press.

KENT, S. (Ed.)

1990 Activity Areas and Architecture: An Interdisciplinary View of the Relationship Between Use of Space and Domestic Built Environments. In: Kent, S. (Ed.) Domestic Architecture and the Use of the Space. Cambridge: Cambridge University Press: $127-152$.

KUS, S.

1983 The Social Representation of Space: Dimensioning the Cosmological and the Quotidian. Moore, J.; Keene, A. (Eds.) Archaeological Hammers and Theory. New York, Academic Press: 278-290.

\section{LE CORBUSIER}

1967 The Modulor. A Harmonious Measure to the Human Scale Universally Applicable to architecture and Mechanics. Londres: Faber y Faber.

LEVI-STRAUSS, C.

1973 Antropología estructural. Buenos Aires: Ed. Universitaria.

LOW, S.M.

1995 Indigenous Architecture and the Spanish American Plaza in Mesoamerica and the Caribbean. American Anthropologist, 97 (4): 748-762.

LYNCH, K.

1966 La imagen de la ciudad. Buenos Aires: Alianza.

MAÑANA BORRAZÁS, P.; BLANCO ROTEA, R; AYLÁN VILA, X.M.

2002 Arqueotectura 1: bases teóricometodológicas para una arqueología de la arquitectura. TAPA, 25.

MERLEAU-PONTY, M.

1962 The Phenomenology of Perception. Cambridge: Harvard University Press

MOORE, J.D.

1996 The Archaeology of Plazas and the Proxemicas of Ritual: Three Andean Traditions. American Anthropologist, 98: 789-802.

NORBERG-SCHULZ, C.

1980 Existencia, espacio y arquitectura. Barcelona: Blume.

PARKER PEARSON, M.; RICHARDS, C. (EDS.)

1994 Architecture and order: approches to social space. Londres: Routledge.

PIAGET, J.

1966 Psicología de la Inteligencia. Buenos Aires: Alianza.

RAGLAN, F.R.

1964 The Temple and the House. Londres: Routledge y Kegan Paul.

RAPOPORT, A.

1969 House Form and Culture. Nueva Jersey: Prentice-Hall.

1972 Vivienda y cultura. Barcelona: Gustavo Gili.

1990 Systems of activities and systems of settings. Kent, S. (Ed.) Domestic architecture and the use of space. Cambridge: Cambridge University Press.

RAUTMANN, A.E.

2000 Population Aggregation, Community Organization, and Plaza-Oriented Pueblos in the American Southwest. Journal of Field Archaeology, 27 (3): 271-283. 
REID, M.L.

1989 A Room With a View: an Examination of Round-Houses, with Particular Reference to Northern Britain. Oxford Journal of Archaeology, 8 (1): 1-40.

SAMSON, R. (Ed.)

1990 The Social Archaeology of Houses. Edimburgo: Edinburgh University Press.

SANDERS, D.

1990 Behaviorable Conventions and Archaeology: Methods for the Analysis of Ancient Architecture. In: Kent, S. (Ed.) Domestic Architecture and the Use of Space. Cambridge, Cambridge University Press: 43-72.

\author{
SCHWARZ, R. \\ 1958 The Church Incarnate. Cambridge: \\ Harvard University Press. \\ SMITH, A. \\ 2001 The Differential Use of Constructed Sacred \\ Space in Southern Britain, from the Late Iron \\ Age to the $4^{\text {th }}$ Century A.D. Oxford: BAR \\ VITRUVIUS \\ British Series 318.
1983 (1931)De Architectura (tradución al inglés de Frank Granger). Cambridge: Harvard University Press. \\ WATSON, O.M. \\ 1970 Proxemic Behaviour. A Cross-Cultural \\ Study. Haia: Mouton.
}

\section{Bibliografía}

\author{
ALLISON, P. M. \\ 1999 The Archaeology of Household Activities. \\ Londres: Routledge.
}

\section{BARTHES, R.}

1986 Semiology and the Urban. In: Gottdiener, M.; Lagopoulos, A. (Eds.) The City and the Sign. Nueva York, Columbia University Press: 88-98.

BERNARDI, $\mathrm{M}$.

1992 Archaeologia del Paesaggio. Edizioni all'insegna del giglio. Florencia: Consiglio Nazionale delle Ricerche, Università degli studi di Siena.

BINFORD, L. (Ed.)

1972 An Archaeologycal Perspective. Nueva York: Academic Press.

BRADLEY, R.

1984 The Sociological Fundations of Prehistoric Britain. Themes and Variations in the Archaeology of Power. Londres: Longmans.

CHILDREN, G.; NASH, G.

1997 Establishing a Discourse: The Language of Landscape. Semiotics of Landscape. Archaeology of Mind. Oxford, Bar International Series 661: 1-4.

CLARKE, D.L.

1968 Analytical Archaeology. Londres: Methuen.

1972 A Provisional Model of an Iron Age Society and Its Settlements System. Clarke, D.L. (Ed.) Models in Archaeology. Londres, Methuen: 801-869.

1977 Spatial Information in Archaeology.
Clarke, D.L. (Ed.) Spatial Archaeology. Londres, Academic Press: 1-32.

CRIADO BOADO, F.

1995 El control arqueológico de obras de trazado lineal: planteamientos desde la arqueología del paisaje. Actas del XXII Congreso Nacional de Arqueología (Vigo, 1993), I. Vigo, Consellería de Cultura/ Concello de Vigo: 253-259.

DELEUZE, G.

1992 Postcript on the Socities of Control. October, 59. Cambridge: 3-8.

ECO, U.

1986 Functionalism and Sign: The Semiotcs of Architecture. Gottdiener, M.; Lagopoulos, A. (Eds.) The City and the Sign. Nueva York, Columbia University Press: 56-85.

1987 How an Exposition Exposes Itself. In: Eco, U. Travels in Hyperreality. Londres, Pan Books: 266-299.

FAHMEL BEYER, B.

1983 The Organization of State-Churches and the Interpretation of Prehistoric Architecture Via Middle Range Theories. Tesis de Maestría. Albuquerque: The University of New México.

GIDDENS, A.

1979 Central Problems in Social Theory: Action Structure and Contradiction in Social Analysis. Londres: Macmillan.

GLASSIE, H.

1975 Folk Housing in Middle Virginia: a 
Structural Analysis of Historic Artifacts.

Knoxville: University of Tennessee Press.

HILLIER, B; HANSON, J.

1984 The Social Logic of Space. Cambridge: Cambridge University Press.

HODDER, I.

1977 Some New Directions in the Spatial Analysis of Archaeological Data at the Regional Scale (macro). In: Clarke, D.L. (Ed.) Spatial Archaeology. Londres: Academic Press: 233-351.

1986 Interpretación en arqueología. Barcelona: Crítica.

HODDER, I; ORTON, C.

1990 Análisis espacial en arqueología. Barcelona: Crítica.

JAMES, M.; JONGEWARD, D.

1975 Nacidos para triunfar. Análisis Transaccional con Experimentos Gestalt. Santiago: Fondo Educativo Interamericano S.A..

JOHNSON, M.

1993 Housing Culture. Traditional Architecture in an English Landscape. Londres: Academy Editions.

LOCOCK, M.

1994 Meaningful Architecture. Locock, M. (Ed.) Meaningful Architecture: Social Interpretations of Buildings. Avebury, Aldershot: 1-13.

MCGUIRE, R.H.; PAYNTER, R. (Eds.)

1991 The Archaeology of Inequality. Oxford: Blackwell.

MILLER, D.; TILLEY, C. (Eds.)

1984 Ideology, Power and Prehistory. Cambridge: Cambride University Press.

PARKES, D.; THRIFT, N.

1978 Putting Time in Its Place. In: Carlstein, D.; Thriff, N. (Eds.) Timing Space and Spacing Time, vol. 1, Make Sense of Time. Londres, E. Arnold: 119-129.

ROSSIGNOL, J.; WANDSNIDER, L.

1992 Space, Time and Archaeological Landscapes. Nueva York: Plenum Press.
SCHIFFER, M.

1972 Archaeology Context and Systemic

Context. American Antiquity, 37: 156-165.

1976 Behavioral Archaeology. Nueva York: Academic Press.

SHACKLE, G.L.S.

1978 Time, Choise and Uncertainty. Carlstein, D.; Thriff, N. (Eds.) Timing Space and Spacing Time, vol. 1, Make Sense of Time. Londres, E. Arnold: 47-55.

SHANKS, M; TILLEY, C.

1987 Social Theory and Archaeology. Cambridge: Polity Press.

STEADMANN, S.

1996 Current Research in the Archaeology of Architecture: Beyond the Foundations. Journal of Archaeological Research, Dordrecht, 4: 51-93.

TILLEY, C.

1994 A Phenomenology of the Landscape: Places, Paths and Monuments. Oxford: Berg.

TOWNSEND, A.

1997 The Materiality: Structured Social Environment of the Maltese Islands During the Temple Building Phase. Semiotics of Landscape. Archaeology of Mind. Oxford, Bar International Series 661: 89. 104.

TUAN, Y.-F.

1977 Space and Place: The Perspective of Experience. Londres. E. Arnold.

UCKO, P.J.; TRINGHAM, R.; DIMBLEY, G.W. (EDS.)

1972 Man, Settlement and Urbanism. Londres: Dackworth.

VEN, C. VAN DE

1981 El espacio en arquitectura. La evolución de una idea nueva en la teoría e historia de los movimientos modernos. Madrid: Cátedra.

WILK, R.; RATHJE, W.

1980 Household Archaeology. American Behavioral Scientist, Nueva York, 25: 617-639. 